\title{
TIP-SEEKING ACTIVE CONTOURS FOR BIOIMAGE SEGMENTATION
}

\author{
Virginie Uhlmann ${ }^{\star}$, Michael Unser ${ }^{\star}$ \\ ^Biomedical Imaging Group, École polytechnique fédérale de Lausanne (EPFL), Switzerland
}

\begin{abstract}
In the present paper, we address the problem of segmenting biological objects featuring corners. The main ingredients of our approach are automated feature-detection methods and mechanisms for introducing kinks in parametric spline snakes. We formulate a novel corner potential that enables the accurate segmentation of objects exhibiting sharp tips or acute angles. The optimization of active contours using the proposed keypoint-based energy yields robuster segmentation results and requires fewer parameters than traditional splinesnake approaches for the same task. The performance of our method is illustrated on microscopic images of two families of Rhabditidse roundworms.
\end{abstract}

Index Terms - Segmentation, bioimage analysis, active contours, keypoints, feature detection, roundworms.

\section{INTRODUCTION}

The ultimate goal of segmentation is the quantification or tracking of objects of interest, making it is an essential step in bioimage analysis $[1,2]$. Active contours, usually referred to as snakes [3], are tools of choice for this task. Active-contour algorithms are traditionally composed of two key elements: the snake model and the snake energy. Many different activecontour models can be found in the literature [4]. We focus in this work on the family of spline snakes [5]. While the model defines the actual snake curve, the energy is a functional to be minimized for the snake to properly segment the objects of interest. Most snake energies are constructed using imagebased information (external energy) or snake-curve properties (internal energy). While many approaches to detect features (or keypoints) exist in the computer-vision community [6], landmark-based segmentation approaches relying on the automated detection of interest points can hardly be found in bioimage informatics where most methods rely instead on points that are defined manually [7].

Our goal in this paper is to make the link between automatic feature detection and active-contour methods in a bioimaging context. To do so, we propose a novel corner potential in combination with the control of tangents offered by Hermite bases [8]. These functions can indeed be used to

This work was funded by the Swiss National Science Foundation under Grants 200020-144355 and 200020-121763. construct a snake model that provides a direct control over the tangent field of the curve. Unlike active contours that generate curves that are smooth by construction, this model has the ability to also represent sharp angles. Hence, it is well-suited to outline biological structures with pointy tips such as worms or protists. Classical spline snakes are smooth by design and require a large number of control points to approximate discontinuous curves. By contrast, the tangent-based snake model is able to perfectly reproduce such features with one control point only. To the best of our knowledge, this is the first time an energy relying on this property is proposed. The corner potential, which unifies automated feature-detection methods and active contours, can be seen as an extension of landmark-based energies. It not only relies on keypoints to attract the snake at corner locations, but also constrains the snake curve to create a sharp angle. Combined with other energy terms, it promotes the robustness of the active contour.

The paper structure is as follows: First, we introduce the tools we rely on to define the corner potential. We then formulate and describe its expression. We illustrate and discuss its capabilities on synthetic images. Finally, we apply our new feature-based energy in two bioimage segmentation problems.

\section{ACTIVE CONTOURS WITH TANGENTIAL CONTROL}

The choice of our snake model is driven by two main considerations. First, we aim at segmenting bioimages, which often feature partially elliptic objects. Relying on a contour model that spans the space of ellipses therefore opens the possibility to generalize the method to various kinds of bio-data. Second, our goal is to capture sharp features, a task that smooth parametric curves can hardly perform.

The active contour with tangential control proposed in [8] meets both requirements as it benefits from ellipsereproducing capabilities and is able to generate sharp transitions. This snake model is schematically represented in Figure 1. Formally, it is defined by an $M$-periodic sequence of anchor points $\{\mathbf{r}[k]\}_{k \in \mathbb{Z}}$ and tangent vectors $\left\{\mathbf{r}^{\prime}[k]\right\}_{k \in \mathbb{Z}}$, where $\mathbf{r}[k]=\mathbf{r}[k+M]=\left.\mathbf{r}(t)\right|_{t=k}$ and $\mathbf{r}^{\prime}[k]=\mathbf{r}^{\prime}[k+M]=\left.\mathbf{r}^{\prime}(t)\right|_{t=k}$. The continuous-domain 


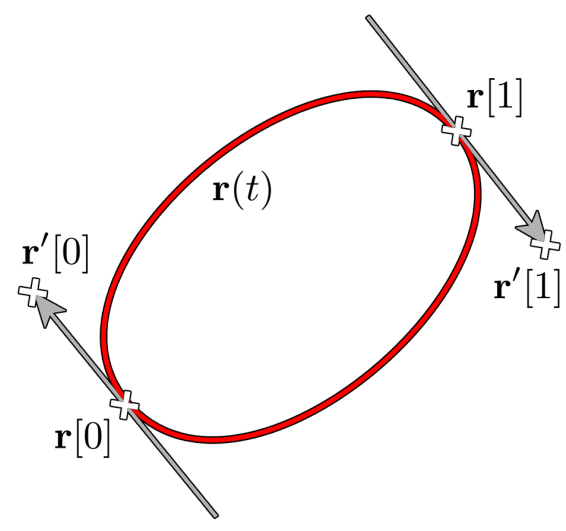

Fig. 1: Spline-snake model with tangential control. The continuous curve $\mathbf{r}(t)$ is defined by a collection of anchor points $\mathbf{r}[k]$ and their associated tangent controls $\mathbf{r}^{\prime}[k]$.

parameterization of the contour is then expressed as

$$
\mathbf{r}(t)=\sum_{k=0}^{M-1}\left(\mathbf{r}[k] \phi_{1, M, \operatorname{per}}(t-k)+\mathbf{r}^{\prime}[k] \phi_{2, M, \operatorname{per}}(t-k)\right)
$$

with $t \in \mathbb{R}$. As the active contour curve is closed, it relies on the $M$-periodized versions of the basis functions

$$
\begin{aligned}
\phi_{1, M, \text { per }}(t) & =\sum_{n=-\infty}^{\infty} \phi_{1, M}(t-M n), \\
\phi_{2, M, \text { per }}(t) & =\sum_{n=-\infty}^{\infty} \phi_{2, M}(t-M n) .
\end{aligned}
$$

The expression of the two exponential Hermite B-splines $\phi_{1, M}$ and $\phi_{2, M}$ is given in [8]. The positive integer $M$ encodes the complexity of the snake shape: while small values of $M$ yield simpler curves, large ones grant more flexibility and generate a richer variety of contours. Increasing the value of $M$ therefore allows one to approximate closed curves of arbitrary complexity at the expense of a larger number of parameters.

\section{KEYPOINT-BASED SNAKE ENERGY}

Algorithms for parametric snakes usually consist of two steps: a rough initialization of the contour followed by an automated optimization to precisely segment its target. The optimal segmentation is found as the minimizer of a cost functional called the snake energy [9]. We now describe our main contribution, a novel feature-based energy that relies on automatically detected corner points.

\subsection{Formulation}

Energies attracting parametric snakes towards a set of userdefined points can be found in the literature [7], as well as methods to drive contour extremities to automatically detected landmarks [10]. We aim at extending this idea by not only imposing automatically detected landmarks to attract the contour, but also to benefit from the direct control on the tangents granted by our snake construction to constrain the local behavior of the curve. In particular, our goal is to derive a corner potential-an energy functional that enables the generation of controlled discontinuities at precise locations on the curve. We propose the feature-based energy

$$
E=\sum_{i=1}^{N_{f}}\left\|\mathbf{r}\left[k_{i}^{*}\right]-\mathbf{f}_{i}\right\|^{2}+M^{2} \frac{\left\|\mathbf{r}^{\prime}\left[k_{i}^{*}\right]\right\|^{2}}{\left\|\mathbf{r}\left[k_{i}^{*}\right]-\mathbf{f}_{i}\right\|^{2}},
$$

where

$$
k_{i}^{*}=\underset{0 \leq k<M}{\operatorname{argmin}}\left\|\mathbf{r}[k]-\mathbf{f}_{i}\right\|^{2} .
$$

The $\mathbf{r}[k]$ and $\mathbf{r}^{\prime}[k]$ are the $M$ control points and tangent vectors in (1), and $\mathbf{f}_{i}$ is the $i$-th element of the $N_{f}$ feature points. Finally, $\|\cdot\|$ denotes the Euclidean norm.

The energy contribution of a given feature point $\mathbf{f}_{i}$ is hence composed of two terms: the squared distance of the control point closest to the feature, and the length of its associated tangent vector divided by the aforementioned distance. In this way, the penalty imposed by the length of the tangent increases as the point approaches the location of a feature, forcing the curve to create a sharp corner. This second term is weighted by $M^{2}$ to impose stronger constraints on the tangents for curves with more control points and, therefore, more degrees of freedom. In practice, a small value is added to the denominator of the second term to avoid numerical difficulties when the feature becomes too close from the control points.

\subsection{Proof of Concept}

In practice, the total energy is formulated as a linear combination of independent energy contributions. We here rely on a synthetic image to demonstrate the benefits of using the keypoint-based energy (2). We study the segmentation of a solid almond-shaped object. As depicted in Figure 2a, the pointy extremities can easily be detected, for instance by a Harris corner detector. This method identifies locations of sudden variation of the gradient in the image. More precisely, it relies on the eigenvalues $\left(\lambda_{1}, \lambda_{2}\right)$ of the Hessian matrix to obtain a corner score $R$ for each point in the image, defined as

$$
R=\lambda_{1} \lambda_{2}-k\left(\lambda_{1}+\lambda_{2}\right) .
$$

The behavior of the detector can thus be tuned by acting on three main parameters. The first one is the so-called sensitivity parameter $k$, for which smaller values allow capturing sharper corners. The second is a minimal value for $R$, which sets a threshold on the accepted quality of corners. Finally, 


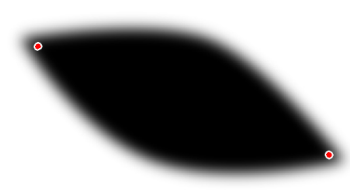

(a)

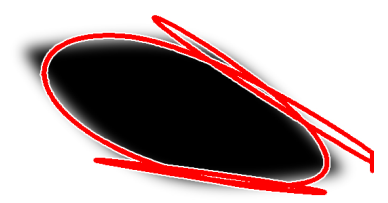

(c)

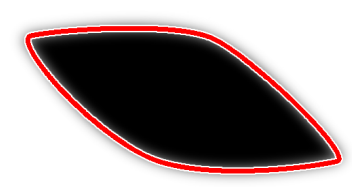

(e)

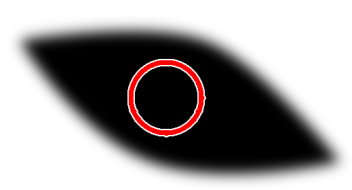

(b)

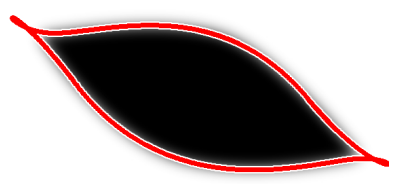

(d)

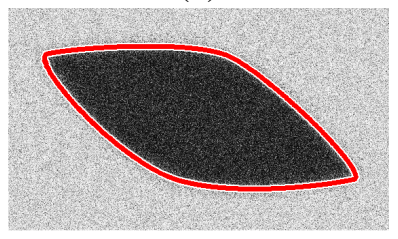

(f)
Fig. 2: Examples of synthetic image. (a) Detected features locations in red; (b) 4-points snake initialization. Segmentation results after optimizing on (c) edge-based energy only; (d) combination of edge- and constraint-based [7] energies; (e) combination of (2) and edge-based energy; (f) Results using a combination of (2) and edge-based energy on the image degraded by additive Gaussian noise.

the support of the filter used to estimate the Hessian matrix can be tuned as well.

When the snake is not encouraged to place control points at shape extremities, it tries to generate a discontinuity by dramatically increasing the length of tangents, resulting in unwanted loops (Figure 2c). Adding a landmark energy that does not involve tangents, such as the one proposed in [7], is still not sufficient (Figure 2d) as it fails to prevent the snake from diverging at the tips of the object. However, constraining both the location of feature points and small tangent values allows for a precise outline of the whole shape, as pictured in Figure 2e. Moreover, both the corner detection method and the snake optimization are robust to noise (see Figure 2f). Results are shown for the same initial conditions depicted in Figure $2 b$ in all four cases.

\section{APPLICATION TO BIOIMAGES}

\subsection{Segmentation of Caenorhabditis Elegans}

We segmented images from the $C$. elegans infection live/dead image set Version 1 provided by F. Ausubel (BBBC10) and available from the Broad Bioimage Benchmark Collection (BBBC, [11]). The living C. elegans worms appear on these images as elongated with pointy extremities and exhibit a va- riety of curved shapes. The difficulty in segmenting these data stems from the need to have a method that is flexible enough to accommodate the sinuous shapes of the worms while retaining the capability to capture sharp features. Sample results are shown in Figure 3.

In Table 1, we report a quantitative comparison of our results against the manually annotated ground truth provided on the BBBC website $^{1}$. A multiscale optimization scheme was used to boost robustness in such potentially crowded environment. Contours are first optimized using the featurebased energy (2) to approach the extremities of the worms, and an edge-based energy is then used to fit the rest of the contour. Our segmentation results are robust to initial conditions provided that the initial snakes do not significantly overlap nearby worms.

Table 1: Jaccard index-Keypoint-driven active contours versus ground truth.

\begin{tabular}{c|ccccccc}
\hline \hline Worm \# & 1 & 4 & 5 & 6 & 10 & 12 & 14 \\
\hline B07 & 0.79 & & 0.83 & 0.79 & & \multirow{2}{*}{0.88} & 0.84 \\
C03 & 0.84 & 0.87 & & & 0.81 & 0.88 \\
\hline \hline
\end{tabular}

\subsection{Segmentation of Heligmosomoides Polygyrus Bakeri}

We also tried our approach on images of unstained Heligmosomoides Polygyrus Bakeri (H. bakeri) worms ${ }^{2}$. This organism, which is a natural parasite of mice and is a purely enteric nematode, appears as translucent and is more difficult to segment than C. elegans. Moreover, as these worms are moving very fast, their extremities appear blurred in most images, although being very pointy in reality. Typical results are shown in Figure 4. We had to initialize snakes closer to the actual contour to get a satisfactory result because of the difficulty of the task.

\section{CONCLUSION}

Our contribution in this paper is the formulation of a corner potential for the optimization of parametric active contours relying on automatically detected features. The proposed energy, inspired from the difficulties encountered in segmenting bioimages, has two main advantages. First, it reduces the sensitivity of snake-based methods to initial conditions or unfavorable imaging settings. Second, it allows the segmentation of objects with sharp corners using a global and flexible model with few parameters. An interesting aspect of the method is that its robustness to noise boils down to the robustness of the feature detector. Similarly, the invariance properties of the detector are inherited by the feature-based energy.

\footnotetext{
${ }^{1}$ In Table 1, the nomenclature corresponds to the ground truth provided at http://www.broadinstitute.org/bbbc/BBBC010/.

${ }^{2}$ Images courtesy of J. Esser, Laboratory of Intestinal Immunology, École polytechnique fédérale de Lausanne (EPFL), Switzerland.
} 

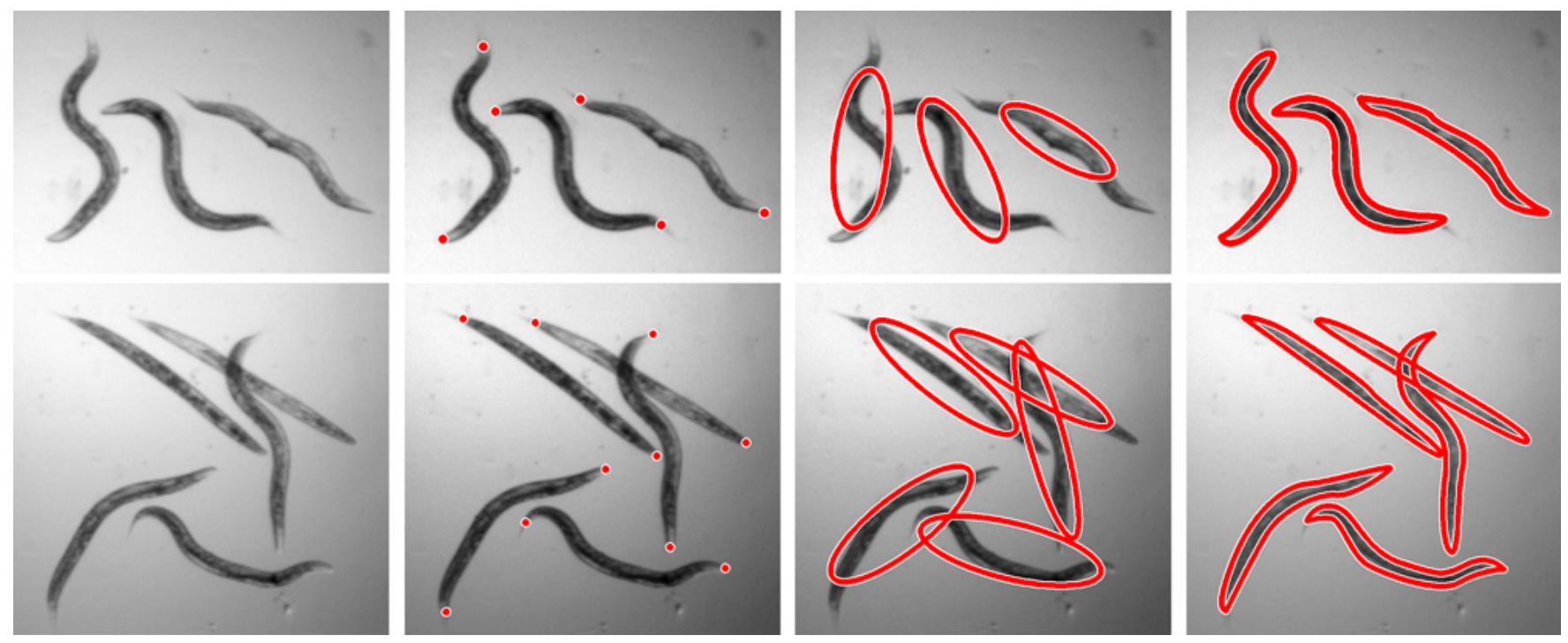

Fig. 3: Caenorhabditis elegans segmentation. Left column: regions of interest from images B07 (top) and C03 (bottom) from the BBBC10 dataset. Center-left: detected keypoints using a Harris detector. Center-right: active-contour initialization. Right: segmentation result after automated optimization.
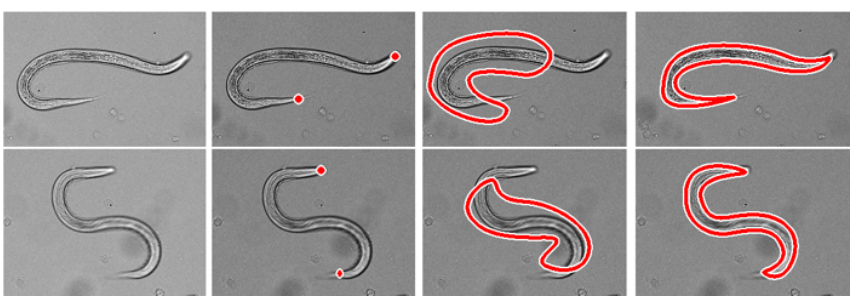

Fig. 4: H. bakeri segmentation. Left column: original images. Center-left: detected keypoints using a Harris detector. Center-right: active-contour initialization. Right: segmentation result after automated optimization.

We first demonstrated the added value of our approach with respect to existing techniques on synthetic data and then illustrated its usefulness for different segmentation tasks on real bioimages. An added benefit of our approach is that the detected objects have a parameterization with landmarks matching the extremities, which facilitates further shape analysis and feature extraction.

\section{REFERENCES}

[1] N. Ray and S.T. Acton, "Active contours for cell tracking," in Proceedings of the Fifth IEEE Southwest Symposium on Image Analysis and Interpretation, Sante Fe, NM, USA, April 7-9, 2002, pp. 274-278.

[2] A. Dufour, V. Meas-Yedid, A. Grassart, and J.-C. Olivo-Marin, "Automated quantification of cell endocytosis using active contours and wavelets," in Proceedings of the Ninetieth IEEE International Conference on Pattern Recognition (ICPR'08), Tampa, FL, USA, December 8-11, 2008, pp. 1-4.

[3] M. Kass, A. Witkin, and D. Terzopoulos, "Snakes: Active contour models," International Journal of Computer Vision, vol. 1, no. 4, pp. 321-331, January 1988.

[4] S. Menet, P. Saint-Marc, and G. Medioni, "Active contour models: Overview, implementation and applications," in Proceedings of the IEEE International Conference on Systems, Man and Cybernetics, Los Angeles, CA, USA, November 47, 1990, pp. 194-199.

[5] P. Brigger, J. Hoeg, and M. Unser, "B-spline snakes: A flexible tool for parametric contour detection," IEEE Transactions on Image Processing, vol. 9, no. 9, pp. 1484-1496, September 2000.

[6] T. Tuytelaars and K. Mikolajczyk, "Local invariant feature detectors: A survey," Foundations and Trends in Computer Graphics and Vision, vol. 3, no. 3, pp. 177-280, January 2008.

[7] M. Jacob, T. Blu, and M. Unser, "Efficient energies and algorithms for parametric snakes," IEEE Transactions on Image Processing, vol. 13, no. 9, pp. 1231-1244, September 2004.

[8] V. Uhlmann, R. Delgado-Gonzalo, C. Conti, L. Romani, and M. Unser, "Exponential Hermite splines for the analysis of biomedical images," in Proceedings of the Thirty-Ninth IEEE International Conference on Acoustics, Speech, and Signal Processing (ICASSP'14), Firenze, Italy, May 4-9, 2014, pp. 1650-1653.

[9] R. Delgado-Gonzalo and M. Unser, "Spline-based framework for interactive segmentation in biomedical imaging," IRBMIngénierie et Recherche Biomédicale / BioMedical Engineering and Research, vol. 34, no. 3, pp. 235-243, June 2013.

[10] O. Henricsson and W. Neuenschwander, "Controlling growing snakes by using key-points," in Proceedings of the Twelfth IEEE International Conference on Pattern Recognition (IAPR'94), Jerusalem, Israel, October 9-13, 1994, vol. 1, pp. 68-73.

[11] V. Ljosa, K.L. Sokolnicki, and A.E. Carpenter, "Annotated high-throughput microscopy image sets for validation," Nature Methods, vol. 9, no. 7, pp. 637, June 2012. 